\title{
MAGIC upper limits to the VHE gamma-ray flux of $3 C 454.3$ in high emission state
}

H. Anderhub ${ }^{1}$, L. A. Antonelli ${ }^{2}$, P. Antoranz ${ }^{3}$, M. Backes ${ }^{4}$, C. Baixeras ${ }^{5}$, S. Balestra ${ }^{3}$, J. A. Barrio ${ }^{3}$, H. Bartko , $^{6}$ D. Bastieri ${ }^{7}$, J. Becerra González ${ }^{8}$, J. K. Becker ${ }^{4}$, W. Bednarek ${ }^{9}$, K. Berger ${ }^{9}$, E. Bernardini ${ }^{10}$, A. Biland ${ }^{1}$,

R. K. Bock ${ }^{6,7}$, G. Bonnoli ${ }^{11}$, P. Bordas ${ }^{12}$, D. Borla Tridon ${ }^{6}$, V. Bosch-Ramon ${ }^{12}$, T. Bretz ${ }^{13}$, I. Britvitch ${ }^{1}$, M. Camara ${ }^{3}$, E. Carmona ${ }^{6}$, S. Commichau ${ }^{1}$, J. L. Contreras ${ }^{3}$, J. Cortina ${ }^{14}$, M. T. Costado ${ }^{8}, i^{5}$, S. Covino $^{2}$, V. Curtef ${ }^{4}$, F. Dazzi $^{7}$, A. De Angelis ${ }^{16}$, E. De Cea del Pozo ${ }^{17}$, R. de los Reyes ${ }^{3}$, B. De Lotto ${ }^{16}$, M. De Maria ${ }^{16}$, F. De Sabata ${ }^{16}$, C. Delgado Mendez ${ }^{8,27}$, A. Dominguez ${ }^{18}$, D. Dorner ${ }^{1}$, M. Doro ${ }^{7}$, D. Elsaesser ${ }^{13}$, M. Errando ${ }^{14}$, D. Ferenc ${ }^{19}$, E. Fernández ${ }^{14}$, R. Firpo ${ }^{14}$, M. V. Fonseca ${ }^{3}$, L. Font ${ }^{5}$, N. Galante ${ }^{6}$, R. J. García López ${ }^{8,15}$, M. Garczarczyk ${ }^{6}$, M. Gaug ${ }^{8}$, F. Goebel ${ }^{6, \star}$, D. Hadasch ${ }^{5}$, M. Hayashida ${ }^{6}$, A. Herrero ${ }^{8,15}$, D. Höhne-Mönch ${ }^{13}$, J. Hose ${ }^{6}$, C. C. Hsu ${ }^{6}$, S. Huber ${ }^{13}$, T. Jogler ${ }^{6}$, D. Kranich ${ }^{1}$, A. La Barbera ${ }^{2}$, A. Laille ${ }^{19}$, E. Leonardo ${ }^{11}$, E. Lindfors ${ }^{20}$, S. Lombardi ${ }^{7}$, F. Longo ${ }^{16}$, M. López ${ }^{7}$, E. Lorenz ${ }^{1,6}$, P. Majumdar ${ }^{10}$, G. Maneva ${ }^{21}$, N. Mankuzhiyil ${ }^{16}$, K. Mannheim ${ }^{13}$, L. Maraschi ${ }^{2}$, M. Mariotti ${ }^{7}$, M. Martínez ${ }^{14}$, D. Mazin ${ }^{14}$, M. Meucci ${ }^{11}$, M. Meyer ${ }^{13}$, J. M. Miranda ${ }^{3}$, R. Mirzoyan ${ }^{6}$, J. Moldón ${ }^{12}$, M. Moles ${ }^{18}$, A. Moralejo ${ }^{14}$, D. Nieto ${ }^{3}$, K. Nilsson ${ }^{20}$, J. Ninkovic ${ }^{6}$, N. Otte ${ }^{6,22,26}$, I. Oya ${ }^{3}$, R. Paoletti ${ }^{11}$, J. M. Paredes ${ }^{12}$, M. Pasanen ${ }^{20}$, D. Pascoli ${ }^{7}$, F. Pauss ${ }^{1}$, R. G. Pegna ${ }^{11}$, M. A. Perez-Torres ${ }^{18}$, M. Persic ${ }^{16,23}$, L. Peruzzo ${ }^{7}$, F. Prada ${ }^{18}$, E. Prandini ${ }^{7}$, N. Puchades ${ }^{14}$, W. Rhode ${ }^{4}$, M. Ribó ${ }^{12}$, J. Rico ${ }^{24,14}$, M. Rissi ${ }^{1}$, A. Robert ${ }^{5}$, S. Rügamer ${ }^{13}$, A. Saggion ${ }^{7}$, T. Y. Saito ${ }^{6}$, M. Salvati ${ }^{2}$, M. Sanchez-Conde ${ }^{18}$, P. Sartori ${ }^{7}$, K. Satalecka ${ }^{10}$, V. Scalzotto ${ }^{7}$, V. Scapin ${ }^{16}$, T. Schweizer ${ }^{6}$, M. Shayduk ${ }^{6}$, K. Shinozaki ${ }^{6}$, S. N. Shore ${ }^{25}$, N. Sidro ${ }^{14}$, A. Sierpowska-Bartosik ${ }^{17}$, A. Sillanpää ${ }^{20}$, J. Sitarek ${ }^{6,9}$, D. Sobczynska ${ }^{9}$, F. Spanier ${ }^{13}$, A. Stamerra ${ }^{11}$, L. S. Stark ${ }^{1}$, L. Takalo ${ }^{20}$, F. Tavecchio ${ }^{2}$, P. Temnikov ${ }^{21}$, D. Tescaro ${ }^{14}$, M. Teshima ${ }^{6}$, M. Tluczykont ${ }^{10}$, D. F. Torres ${ }^{24,17}$, N. Turini ${ }^{11}$, H. Vankov ${ }^{21}$, A. Venturini ${ }^{7}$, V. Vitale ${ }^{16}$, R. M. Wagner ${ }^{6}$, W. Wittek ${ }^{6}$, V. Zabalza ${ }^{12}$, F. Zandanel ${ }^{18}$, R. Zanin ${ }^{14}$, J. Zapatero ${ }^{5}$, S. Vercellone ${ }^{28}$, I. Donnarumma ${ }^{29}$, F. D'Ammando ${ }^{29,30}$, and M. Tavani ${ }^{29,30}$

(Affiliations can be found after the references)

Received 11 November 2008 / Accepted 6 February 2009

\section{ABSTRACT}

Aims. We report upper limits to the very high energy flux $(E>100 \mathrm{GeV})$ of the flat spectrum radio quasar $3 \mathrm{C} 454.3(z=0.859)$ derived by the Cherenkov telescope MAGIC during the high states of July/August and November/December 2007. We compare the upper limits derived in both time slots with the available quasi-simultaneous $\mathrm{MeV}-\mathrm{GeV}$ data from the AGILE $\gamma$-ray satellite and interpret the observational results in the context of leptonic emission models.

Methods. The source was observed with the MAGIC telescope during the active phases of July-August 2007 and November-December 2007 and the data were analyzed with the MAGIC standard analysis tools. For the periods around the ends of July and November, characterized by the most complete multifrequency coverage, we constructed the spectral energy distributions using our data together with nearly simultaneous multifrequency (optical, UV, X-ray and GeV) data.

Results. Only upper limits can be derived from the MAGIC data. The upper limits, once corrected for the expected absorption by the extragalactic background light, together with nearly simultaneous multifrequency data, allow us to constrain the spectral energy distribution of $3 \mathrm{C} 454.3$. The data are consistent with the model expectations based on inverse Compton scattering of the ambient photons from the broad line region by relativistic electrons, which robustly predicts a sharp cut-off above $20-30 \mathrm{GeV}$.

Key words. galaxies: quasars: individual 3C 454.3 - gamma rays: observations - gamma rays: theory

\section{Introduction}

The present list of known extragalactic sources of Very High Energy (VHE; defined here as $E>100 \mathrm{GeV}$ ) radiation includes 24 sources $^{1}$ (e.g. Aharonian et al. 2008a; De Angelis et al. $2008)^{2}$. As expected, the majority of these sources (18) belong

\footnotetext{
* Deceased.

125 , pending the confirmation of the radiogalaxy $3 \mathrm{C} 66 \mathrm{~B}$ detected by MAGIC (Aliu et al. 2009).

${ }^{2}$ See also http://www. mppmu.mpg.de/ rwagner/sources
}

to the high-peaked BL Lac class. The remaining 6 are four low peaked-BL Lac objects (BL Lac: Albert et al. 2007; W Comae: Acciari et al. 2008; S5 0716+71: Teshima et al. 2008; 3C 66A: Swordy et al. 2008), a radiogalaxy (M 87: Aharonian et al. 2003, 2006ba) and a quasar (3C 279: Albert et al. 2008a).

Although the detection of 3C279 indicates that quasars also can, to some extent, emit VHE radiation, general theoretical arguments support the view that, due to internal absorption (e.g. Liu \& Bai 2006; Reimer 2007) and/or to the decrease of the cross section for inverse Compton scattering (e.g. 
Tavecchio \& Ghisellini 2008) powerful flat spectrum radio quasars (FSRQs) cannot be prominent VHE emitters. Moreover, FSRQs are generally located at relatively high redshifts, implying a huge absorption of $\gamma$-rays by the extragalactic background light (EBL). On the other hand, the detection of these sources at VHE would be important for our understanding of their structure and of acceleration/emission mechanisms and would provide a unique opportunity to probe the EBL at relatively high redshifts, allowing us to study its evolution over cosmic time.

3C $454.3(z=0.859)$ is a well known FSRQ, detected several times in the $\gamma$-ray band by the EGRET telescope onboard CGRO, with an average photon index of $\Gamma=2.2$ (Hartmann et al. 1999). In 2005 it underwent a very active phase in optical and X-ray bands, triggering intensive observations in the radio, optical and X-ray (Swift, Chandra, INTEGRAL) bands (Villata et al. 2006; Giommi et al. 2006; Pian et al. 2006). Unfortunately no $\gamma$-ray satellite was operating in the $\mathrm{GeV}$ domain at that time and no information was obtained in that band.

During the summer of 2007, 3C 454.3 was active again, reaching a level of the optical emission comparable to that of 2005. Several observations in the optical, X-ray and $\gamma$-ray band were triggered (optical: KVA, optical-UV: Swift/UVOT, X-ray: Swift/XRT, GeV band: AGILE/GRID). The AGILE satellite (Tavani et al. 2008), still in its science verification phase, detected intense emission from 3C 454.3 (Vercellone et al. 2008a).

Triggered by these observations, the Major Atmospheric Gamma-ray Imaging Cherenkov (MAGIC) Telescope observed 3C 454.3 in July and August 2007. Another $\gamma$-ray active phase was recorded by AGILE in November-December 2007 (Vercellone et al. 2008b, 2009, in prep.), which triggered further observations with MAGIC during that period. In Sect. 2 we describe the MAGIC observations and the analysis procedure. In Sect. 3 we interpret the results in the framework of the widely assumed Synchrotron Self + External Compton (e.g. Sikora et al. 1994) model.

\section{MAGIC observations and data analysis}

MAGIC (Baixeras et al. 2004; Cortina et al. 2005) is a new generation Imaging Atmospheric Cherenkov Telescope at La Palma, Canary Islands, Spain $\left(28.3^{\circ} \mathrm{N}, 17.8^{\circ} \mathrm{W}, 2240 \mathrm{~m}\right.$ asl $)$. Thanks to its low energy trigger threshold of $60 \mathrm{GeV}$, MAGIC is well suited for multiwavelength observations together with the instruments operating in the $\mathrm{GeV}$ range. The parabolically-shaped reflector, with its total mirror area of $236 \mathrm{~m}^{2}$, allows MAGIC to sample a part of the Cherenkov light pool and focus it onto a multi-pixel camera, composed of 576 ultra-sensitive photomultipliers. The total field of view of the camera is $3.5^{\circ}$ and the collection area is of the order of $10^{5} \mathrm{~m}^{2}$ at $200 \mathrm{GeV}$ for a source close to zenith.

The incident light pulses are converted into optical signals, transmitted, via optical fibers and digitized by 2-GHz flash ADCs (Goebel et al. 2007). The primary particle energy and incoming direction are reconstructed by studying the intensity (and area) of the elliptical images and their orientation in the camera. In particular, the shape of the image allows one to suppress hadron-induced showers and thus to reject the hadronic background.

In July and August 2007 observations were carried out in the ON/OFF mode, in which the source was observed on axis (for a total of $9.6 \mathrm{~h}$ ), while for background estimation, observations (for a total of $7.3 \mathrm{~h}$ ) from a region of similar conditions in the sky from where no gamma rays are expected were used. Later, in November and December 2007, additional observations were performed in the false-source tracking (wobble)
Table 1. Derived upper limits on flux for the July and the August 2007 data.

\begin{tabular}{cccccc}
\hline \hline$\langle E\rangle$ & \multicolumn{5}{c}{ U.L. July 17-20 2007 } \\
{$[\mathbf{G e V}]$} & $N_{\mathrm{ON}}$ & $N_{\mathrm{OFF}}$ & Sign. $(\sigma)$ & C.U. & {$\left[\mathrm{erg} \mathrm{cm}^{-2} \mathrm{~s}^{-1}\right]$} \\
\hline 83 & 54188 & 54705 & -1.56 & 0.04 & $0.78 \times 10^{-11}$ \\
186 & 976 & 965 & 0.25 & 0.05 & $0.62 \times 10^{-11}$ \\
476 & 62 & 52.3 & 0.91 & 0.03 & $0.169 \times 10^{-11}$ \\
\hline$\langle E\rangle$ & \multicolumn{5}{c}{ U.L. August 9-22 2007 } \\
{$[\mathbf{G e V}]$} & $N_{\mathrm{ON}}$ & $N_{\text {OFF }}$ & Sign. $(\sigma)$ & C.U. & {$\left[\mathrm{erg} \mathrm{cm}^{-2} \mathrm{~s}^{-1}\right]$} \\
\hline 128 & 5453 & 5539.7 & -0.82 & 0.14 & $2.0 \times 10^{-11}$ \\
186 & 3892 & 3885.1 & 0.078 & 0.03 & $0.3 \times 10^{-11}$ \\
476 & 202 & 220.8 & -0.91 & 0.01 & $0.09 \times 10^{-11}$ \\
\hline
\end{tabular}

The columns represent respectively: the average true energy, the number of ON source events, number of background (OFF) events, the significance, the flux upper limit in Crab Units (C.U.) and in absolute flux units of $\left[\mathrm{erg} \mathrm{cm}^{-2} \mathrm{~s}^{-1}\right.$ ].

Table 2. Derived upper limits on flux for the November and the December 2007 data.

\begin{tabular}{cccccc}
\hline \hline$\langle E\rangle$ & \multicolumn{4}{c}{ U.L. Nov. 27, 30 and Dec. 1 2007 } \\
{$[\mathbf{G e V}]$} & $N_{\mathrm{ON}}$ & $N_{\mathrm{OFF}}$ & Sign. $(\sigma)$ & C.U. & {$\left[\mathrm{erg} \mathrm{cm}^{-2} \mathrm{~s}^{-1}\right]$} \\
\hline 113 & 39900 & 39920 & -0.07 & 0.3 & $4.6 \times 10^{-11}$ \\
235 & 385 & 367 & 0.66 & 0.09 & $0.9 \times 10^{-11}$ \\
\hline
\end{tabular}

The columns represent respectively: the average true energy, the number of ON source events, number of background (OFF) events, the significance, the flux upper limit in Crab Units (C.U.) and in absolute flux units of [erg cm $\mathrm{cm}^{-2} \mathrm{~s}^{-1}$.

mode (Fomin et al. 1994) in which the telescope was pointed alternatingly for $20 \mathrm{~min}$ to two opposite sky positions at $0.4^{\circ}$ offset from the source (total $6.8 \mathrm{~h}$ ). This procedure allows the simultaneous determination of the background and thus no extra OFF observations are needed. The zenith angle of all these observations ranged from 12 to 30 degrees. The weather conditions in July were not as good as those of August, hence the event rate was lower.

The analysis was performed using the standard MAGIC analysis software (Bretz et al. 2005). After calibration and image cleaning based on a two-level tail cut ( 6 photoelectrons for image core and 3 photoelectrons for boundary pixels; see Fegan 1997), the camera images were parameterized by the so-called Hillas image parameters (Hillas 1985). Two additional parameters, namely the time gradient along the main shower axis and the time spread of the shower pixels, were computed (Albert et al. 2008d). Hadronic background suppression was achieved using the Random Forest (RF) method (Breiman 2001; Albert et al. 2008c), in which for each event the so-called "hadronness" is computed, based on the Hillas and the time parameters. The hadronness parameter can be calculated for each event, and is a measure of the probability that the event is not $\gamma$ like. The RF method was also used for the energy estimation. Crab Nebula data from the same periods and zenith angle distributions were studied using the same analysis chain to check the validity of the results.

Since there was no significant signal found, upper limits (95\% CL) were calculated (Rolke et al. 2005) taking into account a $30 \%$ systematic error in energy determination and effective area calculation (see Albert et al. 2008b). Table 1 shows the results for the July-August observations, whereas upper limits for the November-December observation are given in Table 2. 


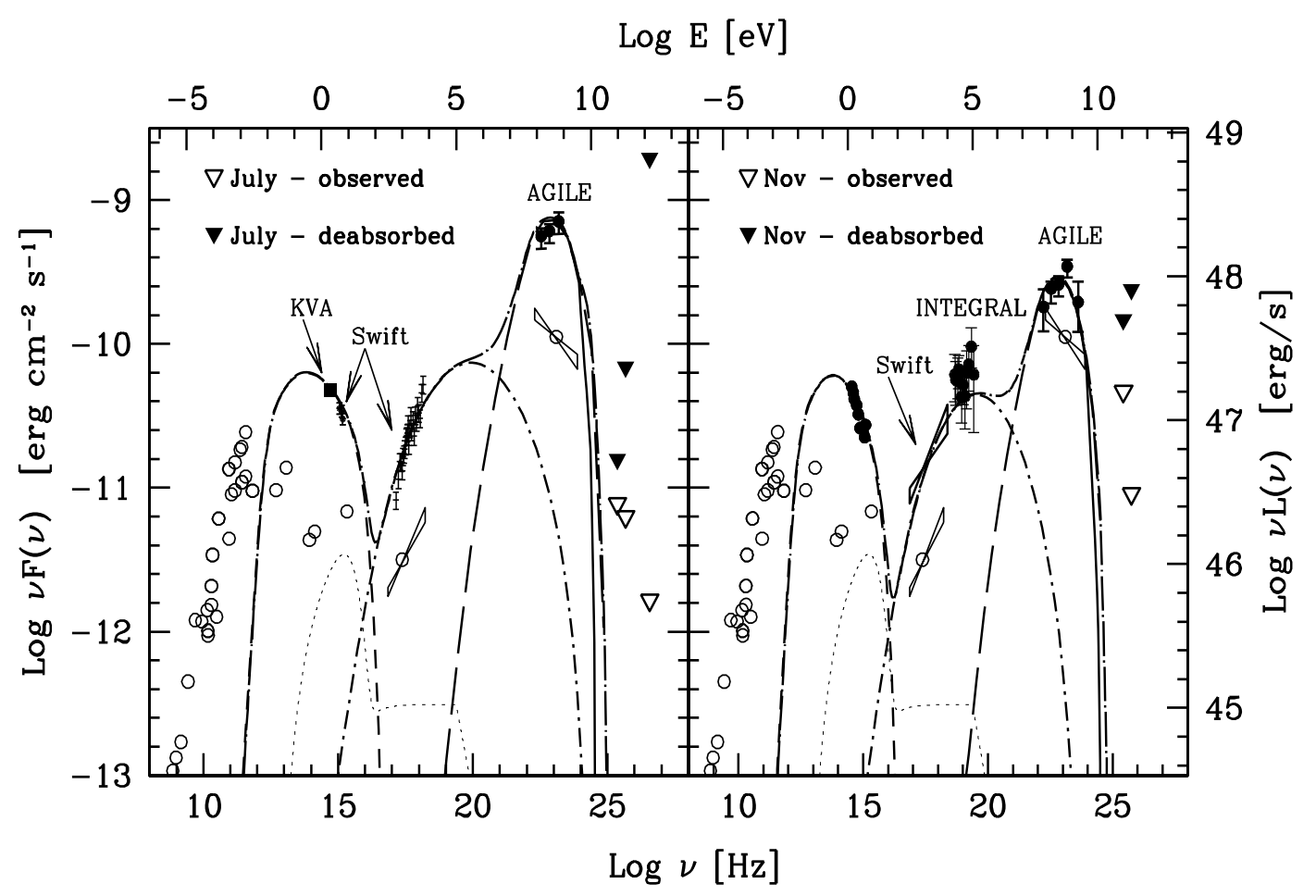

Fig. 1. SED of 3C 454.3 assembled with multifrequency information available for the period close to the MAGIC observation at the end of July 2007 (left panel; optical: KVA, optical-UV: Swift/UVOT, X-ray: Swift/XRT, GeV band: AGILE/GRID) and November 2007 (right panel: opticalUV: Swift/UVOT, X-ray: Swift/XRT and INTEGRAL, GeV band: AGILE/GRID). Triangles report the observed (empty) and the deabsorbed (filled) upper limits of MAGIC in three different bands. For comparison we also report (open circles) historical data (Kuhr et al. 1981; NED, Gear et al. 1994; Stevens et al. 1994; Impey \& Neugebauer 1988; Smith et al. 1988, for radio and optical; Tavecchio et al. 2007, for X-rays from Chandra). The open circle and the bow-tie in the MeV-GeV region indicate the average EGRET spectrum (Hartman et al. 1999). The solid line reports the results of the modelling with the synchrotron-inverse Compton model (see text for details and model parameters). We also report the single emission components: synchrotron (dashed), SSC (dotted-dashed) and EC (long dashed). The dotted line shows the emission of the accretion disk.

\section{Discussion}

The Spectral Energy Distribution (SED) of 3C 454.3 around the epoch of the July and November 2007 MAGIC observations, assembled with the available data, is shown in Fig. 1. For July (left panel) we show the nearly simultaneous data in the optical (KVA, July 24), optical-UV (Swift/UVOT, July 26), X-ray (Swift/XRT, July 26) and $\gamma$-ray (AGILE/GRID) band (average of July 24-30). For November (right panel) the data in the optical, $\mathrm{X}$-ray (Swift/XRT and INTEGRAL) and $\gamma$-ray (AGILE/GRID) data, averaged over the entire period of the AGILE observations (Nov. 11-Dec. 1) are shown. For comparison we also show (open circles) historical data. AGILE/GRID spectra, both for the July and November observations, have been published in Vercellone et al. (2008b).

In the same figure, upper limits from MAGIC observations (18-21 July and 27, 28, 30 November) are shown as triangles (observed: empty; EBL-deabsorbed: filled) (see Table 1). For the EBL deabsorption we used the LowSFR model of Kneiske et al. (2004) which predicts a low level of the EBL close to what is presently inferred from observations, both directly (e.g. Franceschini et al. 2008) and indirectly (Aharonian et al. 2006bb, Mazin \& Raue 2007; Albert et al. 2008a). Note that an even lower level of the EBL (and therefore a lower opacity to $\gamma$-rays) is predicted at the redshift of $3 \mathrm{C} 454.3$ by the model of Primack et al. (2005). However, due to the relatively small difference among the predictions (especially at the frequencies interesting for the absorption of $\gamma$-rays with energies we are considering), the main conclusions of our paper do not critically depend on the specific model used for the absorption.

Figure 1 shows that the (absorption-corrected) MAGIC upper limit at $\sim 100 \mathrm{GeV}$ is inconsistent with the extrapolation of the hard $\gamma$-ray $(100 \mathrm{MeV}-10 \mathrm{GeV})$ spectrum. Therefore, the data indicate there is a break (or a cutoff) of the emission between the $\mathrm{GeV}$ and the $100 \mathrm{GeV}$ band. As discussed below this is consistent with the expectations from the simplest leptonic model.

Emission from blazars is dominated by the non-thermal continuum emitted by a relativistic jet closely aligned towards the observer. The SED of FSRQs is widely interpreted in terms of synchrotron and inverse Compton emission from high-energy electrons. The latter component is probably dominated by the scattering of the external photons (originating in the disk and/or in the broad line region [BLR], Sikora et al. 1994), although the synchrotron self-Compton emission (Maraschi et al. 1992) and the inverse Compton scattering of the direct radiation from the accretion disk (e.g. Dermer \& Schlickeiser 1993) can significantly contribute in the X-ray band. The SED of 3C 454.3, including optical, X-rays and GeV measurements around the end of July, has been discussed and modelled by Ghisellini et al. (2007). However, the model discussed in that work assumes that the $\gamma$-ray spectrum was similar to the average EGRET spectrum, with a soft slope. The spectrum of AGILE (Vercellone et al. 2008b), instead, is rather hard (photon index $\Gamma \simeq 1.7$ in the $100 \mathrm{MeV}-1 \mathrm{GeV}$ band), both for July and November, suggesting a peak of the high-energy component at frequencies above $\sim 10^{23} \mathrm{~Hz}$. The SEDs of November were discussed and modeled in Vercellone et al. (2008b). 
Table 3. Parameters used in the emission model to reproduce the SEDs of 3C 454.3 in July (first row) and November (second row) 2007. Г: bulk Lorentz factor of the emission region; $B$ : magnetic field, in units of $[\mathrm{G}] ; K$ : density of the relativistic electrons, in units of $\left[\mathrm{cm}^{-3}\right] ; n_{1}$ and $n_{2}$ : low and high energy slope of the electron energy distribution; $\gamma_{\min }, \gamma_{b}$ and $\gamma_{\max }$ : minimum energy, break energy and maximum energy of the electron energy distribution; $R$ : radius of the emission region, in units of $\left[10^{15} \mathrm{~cm}\right]$. See text for more details.

\begin{tabular}{ccccccccc}
\hline \hline$\Gamma$ & $B$ & $K$ & $n_{1}$ & $n_{2}$ & $\gamma_{\min }$ & $\gamma_{b}$ & $\gamma_{\max }$ & $R$ \\
\hline 18.4 & 3.1 & $5 \times 10^{5}$ & 1.9 & 3.6 & 85 & 500 & $6.5 \times 10^{3}$ & 6.5 \\
17.8 & 5 & $5 \times 10^{5}$ & 1.9 & 3.9 & 80 & 500 & $3.9 \times 10^{3}$ & 5 \\
\hline
\end{tabular}

To reproduce the multifrequency data we use the emission model fully described in Maraschi \& Tavecchio (2003). Given the focus on the VHE emission, we also consider the absorption of $\gamma$-ray photons through pair production within the BLR. Moreover, the external radiation field (assumed to be isotropic in the frame of the black hole), usually approximated by a black body emission peaking in the UV region, has been calculated using the photoionization code CLOUDY (Ferland et al. 1998). Details on the emission model can be found in Maraschi \& Tavecchio (2003), while the description of the calculation of the external radiation field is reported in Tavecchio \& Ghisellini (2008).

We assume that the emission is produced within a spherical region of radius $R$, in motion with bulk Lorentz factor $\Gamma$. We assume that the corresponding relativistic Doppler factor is $\delta=1 / \Gamma$. The tangled magnetic field has an intensity $B$. The emitting particles, with density $K$, follow a (steady state) brokenpower law energy distribution extending from $\gamma_{\min }$ to $\gamma_{\max }$, with indices $n_{1}$ and $n_{2}$ below and above the break at $\gamma_{b}$. This purely phenomenological distribution has been assumed to reproduce the observed shape of the blazar SEDs, without any specific assumption on the acceleration/cooling mechanism acting on the particles. With this choice we are allowed to assume extreme low-energy slopes $\left(n_{1}<2\right)$ such as those required for 3C 454.3, which cannot be obtained under standard conditions. It is conceivable that, at least in these cases, the electron distribution derives from two different (continuously operating) acceleration mechanisms (see e.g. Sikora et al. 2002). We also neglect the effects related to the cooling of particles in the Klein-Nishina regime, discussed by Moderski et al. (2005). We note, however, that these effects should produce a bump in the opticalUV synchrotron emission which is not apparent in the available data, though the poor coverage does not allow a firm conclusion. We model the external radiation field assuming that the disk emission (dotted line in figure), with a total luminosity of $L_{\text {disk }}=5 \times 10^{46} \mathrm{erg} / \mathrm{s}$, is reprocessed by clouds of the BLR, a sphere with radius $3 \times 10^{17} \mathrm{~cm}$ (we assume that clouds are characterized by standard values of the density $n_{\mathrm{BLR}}=10^{11} \mathrm{~cm}^{-3}$ and hydrogen column density, $N_{\mathrm{H}}=10^{23} \mathrm{~cm}^{-2}$ ). For simplicity, we assume that the distance of the emission region of the jet from the central black hole is smaller than the radius of the BLR, but large enough to neglect the direct disk emission, coming from behind the jet (e.g. Dermer \& Schlickeiser 1993; Vercellone et al. 2008b, consider in the model also this component). To reproduce the shape of the high-energy component, we assume that in the X-ray band the emission is dominated by SSC emission, while EC radiation accounts for the GeV peak.

In our model, the rapid decrease of the flux above a few tens of $\mathrm{GeV}$ would be related to two effects: i) the decrease of the scattering cross section; and ii) the absorption of the produced $\gamma$-rays through pair production. The energy above which the $\mathrm{KN}$ effects become important can be roughly expressed as: $E_{\mathrm{KN}} \simeq 22.5 v_{\mathrm{o}, 15}^{-1} \mathrm{GeV}$, where $v_{\mathrm{o}, 15}$ is the frequency of the external photons (in units of $10^{15} \mathrm{~Hz}$ ). The emission including only the KN effects, neglecting the absorption, is shown by the long dashed-dotted line. The frequency above which the absorption of $\gamma$-rays become effective can be roughly expressed as: $E_{\gamma \gamma} \simeq 60 v_{t, 15}^{-1} \mathrm{GeV}$, where $v_{t, 15}$ is the frequency of the target photons (in units of $10^{15} \mathrm{~Hz}$ ). Therefore, as shown by the solid line in Fig. 1 (calculated including both effects, the internal absorption treated as in Tavecchio \& Mazin 2009), the expected emission above $20-30 \mathrm{GeV}$ is rather small, consistent with the observed upper limits. Note that, although the limit set by KN effects is a characteristic feature of leptonic models, absorption of $\gamma$-rays by soft photons can also be relevant for hadronic models (e.g. Reimer 2007). We also note that the effect of absorption is critically related to the spectrum of the soft target photons. Aharonian et al. (2008b) discussed the case of a black body (or, more generally, a very narrow) soft photon spectrum, showing that in this case very hard $\gamma$-ray spectra could be observed at energies larger than those corresponding to the maximum absorption. In particular, if the black body spectrum peaks in the UV, very hard spectra are expected at energies above $\sim 100 \mathrm{GeV}$. However, as discussed in Tavecchio \& Mazin (2009), realistic BLR spectra are rather broad and the absorption is expected to be important up to energies of $1 \mathrm{TeV}$, hampering the hardening suggested by Aharonian et al. (2008b). For more details on this debated issue see, e.g., Aharonian et al. (2008b), Liu et al. (2008), Sitarek \& Bednarek (2008) and Tavecchio \& Mazin (2009).

Summarizing, we have shown that the upper limits in the VHE band for the 3C 454.3 obtained with the MAGIC telescope are consistent with the expectations of the leptonic models for FSRQs, predicting a sharp decrease of the flux above few tens of $\mathrm{GeV}$, due to the internal absorption of $\gamma$-rays and the decreased efficiency of the inverse Compton emission at high energy. Therefore even upper limits, particularly if accompanied by simultaneous observations in the MeV-GeV band, can be useful to test current emission models for FSRQs. Stronger constraints will definitely be obtained with future multifrequency campaigns already planned with the Fermi Gamma-Ray Telescope.

Acknowledgements. We thank the anonymous referee for comments. We would like to thank the Instituto de Astrofisica de Canarias for the excellent working conditions at the Observatorio del Roque de los Muchachos in La Palma. The support of the German BMBF and MPG, the Italian INFN and Spanish MCINN is gratefully acknowledged. This work was also supported by ETH Research Grant TH 34/043, by the Polish MNiSzW Grant N N203 390834, and by the YIP of the Helmholtz Gemeinschaft. This research has made use of the NASA/IPAC Extragalactic Database (NED) which is operated by the Jet Propulsion Laboratory, California Institute of Technology, under contract with the National Aeronautics and Space Administration.

\section{References}

Acciari, V. A., et al. 2008, ApJ, 685, L73

Aharonian, F., Akhperjanian, A., Beilicke, M., et al. 2003, A\&A, 403, L1

Aharonian, F., Akhperjanian, A. G., Bazer-Bachi, A. R., et al. 2006a, Science, 314,1424

Aharonian, F., Akhperjanian, A. G., Bazer-Bachi, A. R., et al. 2006b, Nature, 440,1018

Aharonian, F., Buckley, J., Kifune, T., \& Sinnis, G. 2008a, Rep. Progr. Phys., 71, 096901

Aharonian, F. A., Khangulyan, D., \& Costamante, L. 2008b, MNRAS, 387, 1206 Albert, J., Aliu, E., Anderhub, H., et al. 2007, ApJ, 666, L17

Albert, J., Aliu, E., Anderhub, H., et al. 2008a, Science, 320, 1752

Albert, J., Aliu, E., Anderhub, H., et al. 2008b, ApJ, 674, 1037

Albert, J., Aliu, E., Anderhub, H., et al. 2008c, Nucl. Instr. Meth. Phys. Res. A, 588, 424 
Albert, J., et al. 2008d, Astropart. Phys., in press [arXiv: 0810. 3586]

Aliu, E., Anderhub, H., Antonelli, L. A., et al. 2009, ApJ, 692, L29

Baixeras, C., Bastieri, D., Bigongiari, C., et al. 2004, Nucl. Instr. Meth. Phys. Res. A, 518, 188

Breiman, L. 2001, Machine Learning, 45, 5

Bretz, T., et al. 2005, in Proc. 29th Int. Cosm. Ray Conf. (Pune, India), 4, 315

Cortina, J., et al. 2005, International Cosmic Ray Conf., 5, 359

De Angelis, A., Mansutti, O., \& Massimo, P. 2008, La Rivista del Nuovo Cimento, 31, 4, 187

Dermer, C. D., \& Schlickeiser, R. 1993, ApJ, 416, 458

Fegan, D. J. 1997, J. Phys. G, 23, 1013

Ferland, G. J., Korista, K. T., Verner, D. A., et al. 1998, PASP, 110, 761

Fomin, V. P., Stepanian, A. A., Lamb, R. C., et al. 1994, Astropart. Phys., 2, 137

Franceschini, A., Rodighiero, G., \& Vaccari, M. 2008, A\&A, 487, 837

Gear, W. K., Stevens, J. A., Hughes, D. H., et al. 1994, MNRAS, 267, 167

Giommi, P., Blustin, A. J., Capalbi, M., et al. 2006, A\&A, 456, 911

Ghisellini, G., Foschini, L., Tavecchio, F., \& Pian, E. 2007, MNRAS, 382, L82

Goebel, F., et al. in Proc. 30th Int. Cosm. Ray Conf. (Merida, Mexico), ed. R. Caballero, et al., 3, 1485

Hartman, R. C., Bertsch, D. L., Bloom, S. D., et al. 1999, ApJS, 123, 79

Hillas, A. M. 1985, Proc. of the 19th ICRC, La Jolla, 3, 445

Kneiske, T. M., Bretz, T., Mannheim, K., \& Hartmann, D. H. 2004, A\&A, 413, 807

Kuehr, H., Pauliny-Toth, I. I. K., Witzel, A., \& Schmidt, J. 1981, AJ, 86, 854

Impey, C. D., \& Neugebauer, G. 1988, AJ, 95, 307

Liu, H. T., \& Bai, J. M. 2006, ApJ, 653, 1089

Liu, H. T., Bai, J. M., \& Ma, L. 2008, ApJ, 688, 148

Maraschi, L., \& Tavecchio, F. 2003, ApJ, 593, 667

Maraschi, L., Ghisellini, G., \& Celotti, A. 1992, ApJ, 397, L5

Mazin, D., \& Raue, M. 2007, A\&A, 471, 439

Moderski, R., Sikora, M., Coppi, P. S., \& Aharonian, F. 2005, MNRAS, 363, 954

Pian, E., Foschini, L., Beckmann, V., et al. 2006, A\&A, 449, L21

Primack, J. R., Bullock, J. S., \& Somerville, R. S. 2005, AIPC, 745, 23

Reimer, A. 2007, ApJ, 665, 1023

Rolke, W. A., López, A. M., \& Conrad, J. 2005, Nucl. Instr. Meth., A551, 493

Sikora, M., Begelman, M. C., \& Rees, M. J. 1994, ApJ, 421, 153

Sikora, M., Błażejowski, M., Moderski, R., \& Madejski, G. M. 2002, ApJ, 577, 78

Sitarek, J., \& Bednarek, W. 2008, MNRAS, in press [arXiv:0807.4228]

Smith, P. S., Elston, R., Berriman, G., Allen, R. G., \& Balonek, T. J. 1988, ApJ, 326, L39

Stevens, J. A., Litchfield, S. J., Robson, E. I., et al. 1994, ApJ, 437, 91

Swordy, S., et al. 2008, The Astronomer's Telegram, 1753

Tavani, M., et al. 2008, A\&A, submitted [arXiv: 0807.4254]

Tavecchio, F., \& Ghisellini, G. 2008, MNRAS, 386, 945

Tavecchio, F., \& Mazin, D. 2009, MNRAS, 392, L40

Tavecchio, F., Maraschi, L., Wolter, A., et al. 2007, ApJ, 662, 900

Tescaro, D., et al., in Proc. 30th Int. Cosm. Ray Conf. (Merida, Mexico), preprint: [arXiv:0709.1410]
Teshima, M., et al. 2008, The Astronomer's Telegram, 1500

Vercellone, S., Chen, A. W., Giuliani, A., et al. 2008a, ApJ, 676, L13

Vercellone, S., Chen, A. W., Giuliani, A., et al. 2008b, ApJ, in press [arXiv: 0809.1737]

Villata, M., Raiteri, C. M., Balonek, T. J., et al. 2006, A\&A, 453, 817

1 ETH Zurich, 8093 Switzerland

2 INAF National Institute for Astrophysics, 00136 Rome, Italy

3 Universidad Complutense, 28040 Madrid, Spain e-mail: Fabrizio.Tavecchio@brera.inaf.it

4 Technische Universität Dortmund, 44221 Dortmund, Germany

5 Universitat Autònoma de Barcelona, 08193 Bellaterra, Spain

6 Max-Planck-Institut für Physik, 80805 München, Germany

7 Università di Padova and INFN, 35131 Padova, Italy

8 Inst. de Astrofísica de Canarias, 38200 La Laguna, Tenerife, Spain

9 University of Łódź, 90236 Lodz, Poland

10 Deutsches Elektronen-Synchrotron (DESY), 15738 Zeuthen, Germany

11 Università di Siena, and INFN Pisa, 53100 Siena, Italy

12 Universitat de Barcelona (ICC/IEEC), 08028 Barcelona, Spain

13 Universität Würzburg, 97074 Würzburg, Germany

14 IFAE, Edifici Cn., Campus UAB, 08193 Bellaterra, Spain

15 Depto. de Astrofisica, Universidad, 38206 La Laguna, Tenerife, Spain

16 Università di Udine, and INFN Trieste, 33100 Udine, Italy

17 Institut de Cienciès de l'Espai (IEEC-CSIC), 08193 Bellaterra, Spain

18 Inst. de Astrofísica de Andalucia (CSIC), 18080 Granada, Spain

19 University of California, Davis, CA-95616-8677, USA

20 Tuorla Observatory, Turku University, 21500 Piikkiö, Finland

21 Inst. for Nucl. Research and Nucl. Energy, 1784 Sofia, Bulgaria

22 Humboldt-Universität zu Berlin, 12489 Berlin, Germany

23 INAF/Osservatorio Astronomico and INFN, 34143 Trieste, Italy

24 ICREA, E-08010 Barcelona, Spain

25 Università di Pisa, and INFN Pisa, 56126 Pisa, Italy

26 now at: University of California, Santa Cruz, CA 95064, USA

27 now at: Centro de Investigaciones Energticas, Medioambientales y Tecnolgicas (CIEMAT), Madrid, Spain

28 INAF/IASF-Milano, via E. Bassini 15, 20133 Milano, Italy

29 INAF/IASF-Roma, via Fosso del Cavaliere 100, 00133 Roma, Italy

30 Dip. di Fisica, Univ. "Tor Vergata", via della Ricerca Scientifica 1, 00133 Roma, Italy 